\title{
Additional Applications of Corneal Cross Linking
}

\author{
Vassilios Kozobolis ${ }^{*}$, Georgios Labiris, Maria Gkika and Haris Sideroudi
}

Eye Institute of Thrace (EIT), Democritus University, Alexandroupolis, Greece

\begin{abstract}
Corneal collagen cross-linking $(\mathrm{CxL})$ is a prevalent surgical method for the management of keratoconus. However, literature suggests that, further to keratoconus, $\mathrm{CxL}$ has a beneficial impact on a series of corneal related diseases and states.

This article attempts to provide a contemporary review for all additional applications of CxL. Specifically, it outlines the most recent studies that demonstrate the beneficial impact of $\mathrm{CxL}$ for iatrogenic ectasias, pellucid marginal degeneration, infectious keratitis, bullous keratitopathy, and for ulcerative keratitis.

The outcome of this review indicates that $\mathrm{CxL}$ could serve as a primary or adjuvant therapeutic modality for all aforementioned corneal-related pathologic states.
\end{abstract}

Keywords: Corneal collagen cross-linking, review, iatrogenic ectasias, pellucid marginal degeneration, bullous keratopathy, infectious keratitis.

\section{INTRODUCTION}

Corneal collagen cross linking is a minimally invasive technique that with the use of riboflavin and Ultraviolet - A irradiation can ensure the production of a stiffening effect capable of augmenting the rigidity of the corneal tissue. The interaction between riboflavin and UVA irradiation results in the formation of bonds between collagen fibrils in the corneal stroma, quasi "freezing" the cornea. The most common use of CXL is the management of ectatic disorders by halting their progression. Nevertheless, a continuous line of new applications is under investigation with promising results.

\section{AFTER REFRACTIVE SURGERY}

It is known that both LASIK and PRK modify corneal stability and overall tissue strength. Researchers suggested that regression after refractive surgery might be reduced by means of CXL. This treatment appears to be particularly applicable to patients who are suspected of having forme fruste keratoconus or those with a correction that exceeds -8 D. These patients may benefit from stiffening of the cornea because keratectasia might not develop.

Early treatments of iatrogenic keratectasia after LASIK performed at the University of Dresden seem to be effective in preventing further progression of the post - refractive surgery ectasia. Hafezi et al. reported that CXL arrested and/or partially reversed keratectasia of 10 patients over a postoperative follow-up of up to 25 months as demonstrated by preoperative and postoperative corneal topography showing a reduction in maximum Keratometry readings [1].

*Address correspondence to this author at the Eye Institute of Thrace, Democritus University, Department of Medicine, Alexandroupolis, Greece; Tel: +302551030990; Fax: 302551039891;

E-mail: vkozompo@med.duth.gr

\section{IN PELLUCID MARGINAL DEGENERATION}

Kymionis et al. performed simultaneous photorefractive keratectomy and CXL in a 34-year-old woman with progressive pellucid marginal corneal degeneration in both eyes. 12 months postoperatively, BCVA improved from $20 / 50$ and $20 / 63$ to $20 / 25$ and $20 / 32$ in the right and left eye, respectively. Corneal topography revealed significant improvement in both eyes [2].

\section{IN BULLOUS KERATOPATHY}

CXL has been shown to have an antiedematous effect on the cornea. Wollensak et al. examined if this effect can be used for the treatment of bullous keratopathy [3]. This clinical interventional case series included 3 patients with bullous keratopathy due to pseudophakia, corneal transplant rejection and Fuchs' endothelial dystrophy. Corneal thickness was reduced. The bullous changes of the epithelium were markedly improved, resulting in loss of pain and discomfort. Visual acuity was significantly improved in the case that did not present prior stromal scarring. In similar results concluded Krueger et al. who performed staged intrastromal delivery of riboflavin with UVA cross-linking in a case of advanced bullous keratopathy [4]. Despite the encouraging results, longer follow-up is necessary to confirm the long-term impact of CXL in bullous keratopathy.

\section{IN INFECTIOUS KERATITIS}

In 2008, Martins et al. [5] demonstrated the antimicrobial properties of CXL against common pathogens. A group of bacteria was tested: Pseudomonas aeruginosa (PA), Staphylococcus aureus (SA), Staphylococcus epidermidis (SE), methicillin-resistant S. aureus (MRSA), multidrugresistant $\mathrm{P}$. aeruginosa (MDRPA), drug-resistant Streptococcus pneumoniae (DRSP), and Candida albicans (CA). Riboflavin/UVA was effective against SA, SE, PA, MRSA, MDRPA, and DRSP, but was ineffective on CA. 
Iseli et al. [6] evaluated the efficacy of CXL for treating infectious melting keratitis. Five patients with infectious keratitis associated with corneal melting were treated with CXL. CXL was performed when the infection did not respond to systemic and topical antibiotic therapy. Follow-up after cross-linking ranged from 1 to 9 months. In all cases, the progression of corneal melting was halted after CXL treatment. Emergency keratoplasty was not necessary in any of the 5 cases presented.

Moreover, Micelli-Ferrari et al. [7] described a case of keratitis caused by the Gram-negative E. Coli treated with CXL with outstanding outcomes.

\section{IN COMPLICATED BULLOUS KERATOPATHY WITH ULCERATIVE KERATITIS}

CXL's antimicrobial and anti-edematous properties were demonstrated by Kozobolis et al. [8] in their report of two patients with combined bullous keratopathy and ulcerative keratitis, resistant to conventional treatments. Both patients presented significant improvement of their visionthreatening corneal ulcer, corneal edema and BCVA for a follow-up period of two months.

\section{CONCLUSION}

CXL is a promising therapeutic intervention for corneal tissue stabilization in diseases that manifest with progressive keratectasia like keratoconus. Moreover, CXL antiedematous and antimicrobial properties were demonstrated in a series of studies suggesting its therapeutic indications in bullous keratopathy and in infectious keratitis as an adjuvant treatment to conventional therapeutic modalities.

\section{REFERENCES}

[1] Hafezi F, Kanellopoulos J, Wiltfang R, Seiler T. Corneal collagen crosslinking with riboflavin and ultraviolet $\mathrm{A}$ to treat induced keratectasia after LASIK. J Cataract Refract Surg 2007; 33(12): 2035-40.

[2] Kymionis GD, Karavitaki AE, Kounis GA, et al. Management of pellucid marginal corneal degeneration with simultaneous customized photorefractive keratectomy and collagen crosslinking. J Cataract Refract Surg 2009; 35(7): 1298-301.

[3] Wollensak G, Aurich H, Wirbelauer C, Pham DT. Potential use of Riboflavin/ UVA crosslinking in bullous keratopathy. Ophthalmic Res 2009; 41(2): 114-7.

[4] Krueger RR, Ramos-Esteban JC, Kanellopoulos AJ. Staged intrastromal delivery of riboflavin with UVA cross-linking in advanced bullous keratopathy: laboratory investigation and first clinical case. J Refract Surg 2008; 24(7): S730-6.

[5] Martins SAR, Combs JC, Noguera G, et al. Antimicrobial efficacy of riboflavin/UVA combination (365 n). In vitro for bacterial and fungal isolates: a potential new treatment for infectious keratitis. Invest Ophthalmol Vis Sci 2008; 49: 3402-8.

[6] Iseli HP, Thiel AM, Hafezi F, et al. Ultraviolet A/riboflavin corneal cross-linking for infectious keratitis associated with corneal melts. Cornea 2008; 27(5): 590-4.

[7] Micelli Ferrari T, Leozappa M, Lorusso M, et al. Escherichia coli keratitis treated with ultraviolet $\mathrm{A} /$ riboflavin corneal cross-linking: a case report. Eur J Ophthalmol 2009; 19(2): 295-7.

[8] Kozobolis V, Labiris G, Gkika M, et al. UVA collagen cross linking treatment of bullous keratopathy combined with corneal ulcer. Cornea 2010; 29(2): 235-8. 